\begin{tabular}{|c|l|}
\hline Title & Barrier effect of collisional processes on electron swarms in nitrogen \\
\hline Author(s) & Sugawara, Hirotake; Satoh, Kohki; Sakai, Y osuke \\
\hline Citation & Journal of Physics D: A pplied Physics, 34(21), 3191-3196 \\
\hline https://doi.org/10.1088/0022-3727/34/21/312 \\
\hline Issue Date & 2001-11-07 \\
\hline Doc URL & http://hdl.handle.net/2115/775 \\
\hline Rights & Copyright (c) 2001 IOP Publishing Ltd. \\
\hline Type & article (author version) \\
\hline File Information & sugawarajpd-2001.pdf \\
\hline
\end{tabular}

Instructions for use 


\title{
Barrier effect of collisional processes on electron swarms in nitrogen
}

\author{
Hirotake Sugawara†, Kohki Satoh $\ddagger$ and Yosuke Sakai† \\ $\dagger$ Division of Electronics and Information Engineering, Graduate School of Engineering, \\ Hokkaido University, Sapporo 060-8628 Japan \\ $\ddagger$ Department of Electrical and Electronic Engineering, Muroran Institute of \\ Technology, Muroran 050-8585 Japan
}

\begin{abstract}
The electron velocity distribution function (EVDF) of electron swarms in $\mathrm{N}_{2}$ has a constricted part at an electron energy of around 2-3 eV. This feature has been explained by considering the barrier effect of vibrational excitation collisions that prevents the gain of energy by slow electrons. In order to investigate the barrier effect in detail further, we simulated the electron behaviour around the energy range of the barrier. We found that momentum transfer collisions also contribute to the barrier effect in collaboration with vibrational excitation. In addition, an evaluation of the electron flow in velocity space is thought to indicate a factor by which the presence of the barrier effect in $\mathrm{N}_{2}$ is revealed in the EVDF; the depression of the electron interchange across the barrier is not only due to the barrier effect itself but also due to the small electron transfer associated with the energy loss by electronic excitation.
\end{abstract}

PACS numbers: 52.80.-s Electric discharges

Published source: Journal of Physics D: Applied Physics, 34(21), 3191-3196 (2001) 


\section{Introduction}

$\mathrm{N}_{2}$ is one of the commonest media of gaseous electronics. Its chemical and kinetic properties have been widely studied for various applications such as atmospheric discharges, the admixture for processing plasmas and as an alternative insulating gas to $\mathrm{SF}_{6}$ for electric power equipment.

In fundamental approaches to $\mathrm{N}_{2}$ applications, the electron energy/velocity distribution function (EEDF/EVDF) is often calculated as an informative quantity which governs the electron-molecule kinetics and from which electron transport parameters can be derived. An interesting feature of the EEDF/EVDF in $\mathrm{N}_{2}$ is that it has a constricted part at an electron energy of around 2-3 eV (e.g., Taniguchi et al 1978, Pitchford and Phelps 1982, etc). The mechanism that induces this appearance has been explained by considering the effect of the electron energy loss by vibrational excitation. Engelhardt et al (1964) noted that the vibrational excitation collision cross section acts as a barrier that prevents the energy gain of slow electrons. Garscadden and Nagpal (1995) and Slavík and Colonna (1997) also mentioned this effect. However, in spite of many remarks, a focused discussion on this effect has rarely been made.

In this paper, we investigate the barrier effect of collisional processes in $\mathrm{N}_{2}$. The behaviour of electrons around an energy range concerned with the barrier effect is simulated. Furthermore, we evaluate the electron interchange across the barrier by considering the electron flow in velocity space to show a factor by which the presence of the barrier effect in $\mathrm{N}_{2}$ reveals itself in the EVDF.

\section{Simulation methods and conditions}

A propagator method (PM), which is a computational technique to solve the Boltzmann equation, is employed for the present EVDF calculation. In the PM, velocity space is divided into cells, and intercellular electron flow representing the ballistic flight of electrons under an electric field and the electron scattering by gas molecules is calculated 
by a transition operator called the propagator (e.g. Sugawara et al 1997, 1998). The initial electrons are released with a Maxwellian EVDF with a mean electron energy of $1 \mathrm{eV}$. The equilibrium EVDF is obtained after a time long enough for the electron swarm relaxation (approximately tens of nanoseconds with the present conditions). In addition to the PM, a Monte Carlo simulation (MCS) is also performed to verify the PM result and to observe single electron motion. More than 100000 electrons are sampled in the MCS.

The present set of the electron collision cross sections of $\mathrm{N}_{2}$ is taken from Ohmori et al (1988). This set consists of the cross sections of momentum transfer $\left(q_{\mathrm{mom}}\right)$, ionization $\left(q_{\mathrm{ion}}\right)$, dissociation $\left(q_{\mathrm{dis}}\right)$, vibrational excitation $\left(q_{\mathrm{vib}, j}, 1 \leq j \leq 10\right)$, and electronic excitation $\left(q_{\mathrm{ex}, j}, 1 \leq j \leq 19\right)$. They are shown in figure 4 . The number density $N$ of $\mathrm{N}_{2}$ molecules is set to be $3.54 \times 10^{16} \mathrm{~cm}^{-3}$ as the value at $133 \mathrm{~Pa}$ (1 Torr) at $0{ }^{\circ} \mathrm{C}$. Isotropic scattering is assumed for all the collisions.

Assuming a uniform electric field $\boldsymbol{E}$ is applied, spherical coordinates $(v, \theta, \phi)$ for the electron velocity vector $\boldsymbol{v}$ are defined; $v=|\boldsymbol{v}|, \theta$ is the angle between $\boldsymbol{v}$ and $-\boldsymbol{E}$, and $\phi$ is the azimuth. The primitive form of the EVDF, $f(\boldsymbol{v})=f(v, \theta, \phi)$, having a dimension of (speed $)^{-3}$ is defined under the following normalization condition:

$$
\int_{\boldsymbol{v}} f(\boldsymbol{v}) \mathrm{d} \boldsymbol{v}=\int_{v=0}^{\infty} \int_{\theta=0}^{\pi} \int_{\phi=0}^{2 \pi} f(v, \theta, \phi) v^{2} \sin \theta \mathrm{d} v \mathrm{~d} \theta \mathrm{d} \phi=1
$$

Note that the calculation results of the EVDF in the next section are presented in integrated forms as functions of the velocity components concerned.

The reduced electric field $E / N$ is chosen to be $100-400 \mathrm{Td}\left(1 \mathrm{Td}=10^{-17} \mathrm{~V} \mathrm{~cm}^{2}\right)$. In this $E / N$ range, the EVDF apparently indicates the presence of the barrier effect. In particular, we will analyse in detail the EVDF at $200 \mathrm{Td}$ as a characteristic example. 


\section{Results and discussion}

\subsection{Electron speed distribution}

Figure 4 shows the electron speed distribution $f(v)$ as a function of the random speed $v=|\boldsymbol{v}|$. Here, $v$ is plotted with a scale of $v_{1}=5.93 \times 10^{7} \mathrm{~cm} \mathrm{~s}^{-1}$ (the electron speed associated with $1 \mathrm{eV}$ ), so that one can easily convert $v$ to the corresponding electron energy $\epsilon$ in eV by using $\epsilon=\epsilon_{1}\left(v / v_{1}\right)^{2}$ with $\epsilon_{1}=1 \mathrm{eV}$. The dimension of $f(v)$ is (speed $)^{-1}$, and $f(v)$ is derived from $f(v, \theta, \phi)$ as follows:

$$
f(v)=\int_{\theta=0}^{\pi} \int_{\phi=0}^{2 \pi} f(v, \theta, \phi) v^{2} \sin \theta \mathrm{d} \theta \mathrm{d} \phi .
$$

The presence of the barrier effect is thought to be indicated by the dip in $f(v)$ around $v=\sqrt{3} v_{1}(\epsilon=3 \mathrm{eV})$ where $q_{\mathrm{vib}}$ lies. The conventional explanation for the barrier effect in the literature focuses on the energy-loss process of vibrational excitation. On the other hand, little attention has been given to the role of the $q_{\text {mom }}$ peak, although the $q_{\text {mom }}$ peak is about three times as high as that of $q_{\mathrm{vib}}$. We will examine the contribution of the $q_{\text {mom }}$ peak to the barrier effect later.

Here, for a comparison or background of the discussion which follows, let us refer to similar effects in Ar and HCl. Makabe and Mori (1982) showed a two-peaked EEDF in Ar at low $E / N$ that lies over the Ramsauer-Townsend minimum (RTM) of $q_{\text {mom }}$. Being interested in this result, Suzuki et al (2001) simulated the electron behavior around the RTM by an MCS and explained that the electrons accelerating over the RTM are scattered back by increasing $q_{\text {mom }}$ on the higher-energy side of the RTM. Penetrante and Bardsley (1983) also showed such an EVDF in $\mathrm{HCl}$. It can be pointed out for $\mathrm{HCl}$ that $q_{\text {mom }}$ of $\mathrm{HCl}$ has a dip like Ar and $q_{\text {vib }}$ lies over the energy range of the dip. Since $\mathrm{HCl}$ also has attachment and rotational excitation processes, further analysis is to be left to future work elsewhere.

Let us now return to the EVDF in $\mathrm{N}_{2}$. The $\operatorname{EVDF} f(\boldsymbol{v})=f(v, \theta, \phi)$ is uniform for $\phi$ and dependent on $\theta$. The dependence of the EVDF on $\theta$ is often represented by the Legendre polynomial expansion terms $f_{n}(v)$ of the spherical harmonics. With the 
$n$ th-order Legendre polynomial $P_{n}, f(v, \theta, \phi)$ is expanded as

$$
\begin{aligned}
& 4 \pi v^{2} f(v, \theta, \phi)=\sum_{n=0}^{\infty} P_{n}(\cos \theta) f_{n}(v) \\
& f_{n}(v)=(2 n+1) \int_{\theta=0}^{\pi} \int_{\phi=0}^{2 \pi} P_{n}(\cos \theta) f(v, \theta, \phi) v^{2} \sin \theta \mathrm{d} \theta \mathrm{d} \phi .
\end{aligned}
$$

Figure 4 shows $f_{n}(v)$ for $n=0,1,2$ calculated by the PM and MCS at 200 Td. Terms $f_{0}(v)$ (identical to $\left.f(v)\right)$ and $f_{1}(v)$ are the isotropic and directional components, respectively, and $f_{2}(v)$ represents the concentration of $\boldsymbol{v}$ to the directions of $\theta=0$ and $\pi$. By introducing a ratio $f_{1} / f_{0}$ to quantify the anisotropy of the EVDF, we see that $f_{1} / f_{0}$ has a peak near the beginning of the energy range in which the $q_{\text {mom }}$ and $q_{\text {vib }}$ peaks lie. Here, let us define 'barrier region' as being the energy range of $2-3 \mathrm{eV}$ for the following discussions. The decrease in $f_{1} / f_{0}$ with $v$ indicates that the EVDF is made less anisotropic by scattering of electrons during their acceleration across the barrier region.

\subsection{Caging of slow electrons}

In order to depict the distribution of $\boldsymbol{v}$ more visually with information of its direction, a two-component form of the EVDF, $f\left(v_{\|}, v_{\perp}\right)$, is shown in figure 4 . Figure 4 is a contour plot of figure 4. Here, $f\left(v_{\|}, v_{\perp}\right)$ is related to $f(v, \theta, \phi)$ as

$$
f\left(v_{\|}, v_{\perp}\right)=\int_{0}^{2 \pi} f(v, \theta, \phi) v \sin \theta \mathrm{d} \phi
$$

where $v_{\|}=v \cos \theta, v_{\perp}=v \sin \theta$ and $f\left(v_{\|}, v_{\perp}\right)$ satisfies

$$
\int_{v_{\|}=-\infty}^{\infty} \int_{v_{\perp}=0}^{\infty} f\left(v_{\|}, v_{\perp}\right) \mathrm{d} v_{\|} \mathrm{d} v_{\perp}=1
$$

In figure 4, the broken semicircle indicates the upper end of the barrier region at $\epsilon=3 \mathrm{eV}$. When considering the barrier in three-dimensional velocity space, it becomes a spherical cage for slow electrons. The valley between the two peaks locates over the semicircle with a shift toward the $+v_{\|}$direction, and the peaks also shift in the same direction. This shift is understood from the electron flow in velocity space illustrated in figure 4 . Electrons are accelerated by $\boldsymbol{E}$ towards the $+v_{\|}$direction as 
$\left(\mathrm{d} v_{\|} / \mathrm{d} t, \mathrm{~d} v_{\perp} / \mathrm{d} t\right)=(e E / m, 0)$, where $e$ and $m$ are the electronic charge and mass, and the barrier scatters them. An MCS result of single electron motion in the cage is presented in figure 4 as an example of these processes. The shift of the valley mentioned above suggests that the electron concentration decays by scattering during the electron penetration into the barrier region and the barrier blockades not only the outward flow from the cage but also the inward flow to the cage.

In order to examine the barrier effect of the $q_{\text {mom }}$ peak, the number of collisions that an electron experiences until it escapes from the cage was counted by an additional MCS. An electron is released with an initial energy of $0.1 \mathrm{eV}$, and traced until $\epsilon$ exceeds a given limit energy. This MCS was performed for three sets of the electron collision cross sections. The first one is of $\mathrm{N}_{2}$ unchanged from Ohmori et al (1988) (case 1). The others are partly modified sets; the second one has $q_{\text {mom }}$ truncated at $10^{-15} \mathrm{~cm}^{2}$ (case 2), and the third one is without $q_{\text {vib }}$ (case 3).

Figure 4 shows the average counts $n_{\text {mom }}$ and $n_{\text {vib }}$ of electron collisions in the interactions with $q_{\mathrm{mom}}$ and $q_{\mathrm{vib}}$, respectively. In case 1 (full curve), $n_{\text {mom }}$ has a large gradient locally in the barrier region because of the $q_{\text {mom }}$ peak. Without this peak (case 2, broken curve), electrons can escape from the cage with less collisions than in case 1 . Here, it is to be noted that $n_{\text {vib }}$ in case 2 also becomes smaller than in case 1 although $q_{\text {vib }}$ is unchanged. The absence of the $q_{\text {mom }}$ peak shortens the residence time of the electrons in the cage, which decreases $n_{\text {vib }}$. When $q_{\text {vib }}$ is missing instead of the $q_{\text {mom }}$ peak (case 3 , dotted curve), the presence of the barrier effect is no longer indicated. These results suggest that the barrier effect in $\mathrm{N}_{2}$ originates mainly in $q_{\mathrm{vib}}$ and is supported by the $q_{\text {mom }}$ peak. The $q_{\text {mom }}$ peak holds slow electrons in the cage, which gives the caged electrons more opportunity of vibrational excitation collisions.

\subsection{The electron flow balance}

The caged electrons discussed above will eventually escape from the cage, and will return into the cage when they lose their energies by inelastic collisions. These processes form 
an electron circulation in velocity space. Let us derive a balance equation for the electron flow in equilibrium. This will explain why in $\mathrm{N}_{2}$ the presence of the barrier effect is apparently indicated in the EVDF.

A spherical boundary $|\boldsymbol{v}|=v$ is considered in three-dimensional velocity space. The electron flow across the boundary can be analysed as being divided into the following three components (see figure 4 ). The acceleration flow $\Phi_{\text {acc }}$ is defined as the number of electrons per unit time that pass through the boundary outwards under the action of $\boldsymbol{E}$. The slowdown flow $\Phi_{\text {slow }}$ represents those passing through the boundary inwards. The electron transfer $\Phi_{\text {inel }}$ is of those diving to the inside region of the boundary from the outside by energy loss due to inelastic collisions. They are given as follows:

$$
\begin{aligned}
& \Phi_{\text {acc }}(v)=\int_{\theta=0}^{\pi / 2} \int_{\phi=0}^{2 \pi} \frac{e E}{m} \cos \theta f(v, \theta, \phi) v^{2} \sin \theta \mathrm{d} \theta \mathrm{d} \phi \\
& \Phi_{\text {slow }}(v)=\int_{\theta=\pi / 2}^{\pi} \int_{\phi=0}^{2 \pi} \frac{e E}{m}(-\cos \theta) f(v, \theta, \phi) v^{2} \sin \theta \mathrm{d} \theta \mathrm{d} \phi \\
& \Phi_{\text {inel }}(v)=\sum_{j} \int_{v}^{\left(v^{2}+v_{1}^{2} \epsilon_{j} / \epsilon_{1}\right)^{1 / 2}} N q_{j}\left(v^{\prime}\right) v^{\prime} f\left(v^{\prime}\right) \mathrm{d} v^{\prime}+2 \Phi_{\text {ion }}(v) \\
& \Phi_{\text {ion }}(v)=\int_{v}^{\infty} N q_{\text {ion }}\left(v^{\prime}\right) v^{\prime} f\left(v^{\prime}\right) \frac{\min \left[\left(v / v_{1}\right)^{2} \epsilon_{1},\left(v^{\prime} / v_{1}\right)^{2} \epsilon_{1}-\epsilon_{\text {ion }}\right]}{\left(v^{\prime} / v_{1}\right)^{2} \epsilon_{1}-\epsilon_{\text {ion }}} \mathrm{d} v^{\prime}
\end{aligned}
$$

where $q_{j}$ is the $j$ th cross section among $q_{\mathrm{vib}}, q_{\mathrm{ex}}$ and $q_{\mathrm{dis}}$, and $\epsilon_{j}$ is the corresponding loss energy. $\Phi_{\text {ion }}$ represents the electron supply to the inside of the boundary by ionization. It is assumed for ionization that the residual energy of a primary electron is divided into two parts for the primary and secondary electrons at a ratio $\Delta:(1-\Delta)$, and $\Delta$ is distributed from 0 to 1 uniformly. Energy loss due to elastic collisions and energy gain by superelastic collisions are ignored.

From the electron conservation inside of the boundary, we obtain

$$
\begin{aligned}
R_{\text {ion }} \int_{0}^{v} f\left(v^{\prime}\right) \mathrm{d} v^{\prime}= & \int_{0}^{v} N q_{\text {ion }}\left(v^{\prime}\right) v^{\prime} f\left(v^{\prime}\right) \mathrm{d} v^{\prime} \\
& -\Phi_{\text {acc }}(v)+\Phi_{\text {slow }}(v)+\Phi_{\text {inel }}(v)
\end{aligned}
$$

where $R_{\text {ion }}$ is the ionization frequency which is defined as

$$
R_{\text {ion }}=\int_{0}^{\infty} N q_{\text {ion }}(v) v f(v) \mathrm{d} v
$$


When we consider electron attachment in electro-negative gases, $q_{\text {ion }}$ and $R_{\text {ion }}$ in equation (11) are replaced respectively with $q_{\text {ion }}-q_{\text {att }}$ and $R_{\text {ion }}-R_{\text {att }}$ to consider the effective ionization. In $\mathrm{N}_{2}$ at $200 \mathrm{Td}$, the terms related to ionization are negligible compared to the others. Then, equation (11) yields

$$
\Phi_{\text {acc }}(v)=\Phi_{\text {slow }}(v)+\Phi_{\text {inel }}(v) .
$$

The PM results of $\Phi_{\text {acc }}, \Phi_{\text {slow }}$ and $\Phi_{\text {inel }}$ shown in figure 4 satisfy this relation with a relative difference of about $0.5 \%$. Figure 4 also shows the shift of the valley observed in figure 4 , i.e. the dip in $\Phi_{\text {acc }}$ locates at a higher $v$ than that of $\Phi_{\text {slow }}$.

When an inelastic energy-loss process for an electron occurs, the value of $v$ of the electron changes instantly. For a transition $v^{\prime} \rightarrow v$, this is regarded as an electron disappearance at $v^{\prime}$ and an electron appearance at $v$ in velocity space. Figure 4 shows the distributions of such $v^{\prime}$ and $v . \Psi_{\text {out,vib }} \mathrm{d} v$ and $\Psi_{\text {out,ex }} \mathrm{d} v$ represent the numbers of electron disappearance per unit time due to vibrational and electronic excitations, respectively, and $\Psi_{\mathrm{in}, \mathrm{vib}} \mathrm{d} v$ and $\Psi_{\mathrm{in}, \mathrm{ex}} \mathrm{d} v$ are those for electron appearance. Their values are given by taking the sum of the following terms for corresponding $j$ :

$$
\begin{aligned}
& \Psi_{\mathrm{out}, j}(v)=N q_{j}(v) v f(v) \\
& \Psi_{\mathrm{in}, j}(v)=N q_{j}\left(v_{j}^{\prime}\right) v_{j}^{\prime} f\left(v_{j}^{\prime}\right)\left(v / v_{j}^{\prime}\right)
\end{aligned}
$$

where $v_{j}^{\prime}=\left(v^{2}+v_{1}^{2} \epsilon_{j} / \epsilon_{1}\right)^{1 / 2}$. They are related to $\Phi_{\text {inel }}$ as

$$
\Phi_{\text {inel }}(v)=\sum_{j} \int_{0}^{v}\left[\Psi_{\text {in }, j}\left(v^{\prime}\right)-\Psi_{\text {out }, j}\left(v^{\prime}\right)\right] \mathrm{d} v^{\prime} .
$$

From figure 4 , we see that $\Psi_{\text {in,ex }}<\Psi_{\text {in,vib }}$ for $v<\sqrt{3} v_{1}$, i.e., the number of electrons transferred into the cage by electronic excitation is small compared to those concerned with vibrational excitation. This is also suggested in figure 4 by the dip of $\Phi_{\text {inel }}$ around $v=2 v_{1}$. The depression of the electron interchange across the barrier is not only due to the barrier effect itself but also due to the small electron transfer into the cage associated with electronic excitation. This mutual isolation is thought to be a factor of the mechanism by which the barrier effect emerges as the constriction of the EVDF. 


\subsection{The first-order Legendre expansion term and inelastic energy loss}

When comparing figures 4 and 4 , one may notice that the shapes of $\Phi_{\text {inel }}(v)$ and $f_{1}(v)$ resemble each other. In fact, we can derive a relation $\Phi_{\text {inel }} \propto f_{1}$ from equations $(7),(8)$ and (13) as

$$
\begin{aligned}
\Phi_{\text {inel }}(v) & =\Phi_{\text {acc }}(v)-\Phi_{\text {slow }}(v) \\
& =\int_{\theta=0}^{\pi} \int_{\phi=0}^{2 \pi} \frac{e E}{m} \cos \theta f(v, \theta, \phi) v^{2} \sin \theta \mathrm{d} \theta \mathrm{d} \phi \\
& =\frac{1}{3} \frac{e E}{m} f_{1}(v) .
\end{aligned}
$$

We find here that $f_{1}(v)$ is directly related to the electron transfer across $v$ associated with the inelastic energy loss.

This fact can also be understood from the viewpoint of the energy balance of an electron swarm. The normalized energy gain per unit time, $\epsilon_{\text {gain }}$, is represented with $f_{1}$ through the drift velocity $W$ as

$$
\epsilon_{\text {gain }}=e E W=e E\left(\frac{1}{3} \int_{0}^{\infty} v f_{1}(v) \mathrm{d} v\right)
$$

On the other hand, the corresponding energy loss, $\epsilon_{\text {loss }}$, is given from an integral for $\Psi_{\text {in }}$ and $\Psi_{\text {out }}$ with a weight of the kinetic energy $\frac{1}{2} m v^{2}$ :

$$
\begin{aligned}
\epsilon_{\mathrm{loss}} & =-\int_{0}^{\infty} \frac{1}{2} m v^{2} \sum_{j}\left[\Psi_{\text {in }, j}(v)-\Psi_{\text {out }, j}(v)\right] \mathrm{d} v \\
& =-\int_{0}^{\infty} \frac{1}{2} m v^{2} \frac{\mathrm{d}}{\mathrm{d} v} \Phi_{\text {inel }}(v) \mathrm{d} v .
\end{aligned}
$$

The energy balance equation $\epsilon_{\text {gain }}=\epsilon_{\text {loss }}$ is obtained from the integral over $v$ of equation (17) with a factor $m v$.

Here, equation (17) can also be rewritten as follows by taking its derivative after substitution of equations (14)-(16) for $\Phi_{\text {inel }}$ :

$$
\frac{1}{3} \frac{e E}{m} \frac{\mathrm{d}}{\mathrm{d} v} f_{1}(v)=\sum_{j} N q_{j}(v) v f(v)-\sum_{j} N q_{j}\left(v_{j}^{\prime}\right) v_{j}^{\prime} f\left(v_{j}^{\prime}\right)\left(v / v_{j}^{\prime}\right) .
$$

This is a specific form of the formula relating $f_{0}$ and $f_{1}$ in the Legendre expansion from Boltzmann equation analysis (e.g. equation (21) in Holstein (1946)). What $\Phi_{\text {inel }}$ 
represents is considered as a physical interpretation to the mathematical result of the Legendre expansion.

\section{Conclusions}

We have analyzed the barrier effect of electron-molecule collisions on electron swarms in $\mathrm{N}_{2}$. In addition to the effect of vibrational excitation, from which the barrier effect has been mainly explained, the effect of momentum transfer collisions was also examined. By simulating the electron behaviour around the $q_{\text {vib }}$ and $q_{\text {mom }}$ peaks, we have shown that the $q_{\text {mom }}$ peak also contributes to the barrier effect in collaboration with $q_{\text {vib }}$. Momentum transfer collisions have the effect of holding slow electrons in the low-speed region surrounded by the $q_{\text {mom }}$ peak, which gives the slow electrons more opportunity of losing energy by vibrational excitation collisions.

In the analysis of the electron flow in velocity space, it was shown that the number of electrons transferred into the low-speed region by electronic excitation is small compared to those concerned with vibrational excitation. The depression of the electron interchange across the barrier is not only due to the barrier effect itself, but also due to the small inward electron transfer associated with electronic excitation. This mutual isolation is thought to be a factor of the mechanism by which the barrier effect emerges as the constriction of the EVDF.

We have also presented a relation between the directional component $f_{1}(v)$ of the EVDF expanded in terms of the Legendre polynomials and the electron transfer in velocity space associated with inelastic energy loss. This relation was found to be a specific form of the formula relating $f_{0}$ and $f_{1}$ in the Legendre expansion from Boltzmann equation analysis. Through a quantification of the inelastic electron transfer, we have given a physical interpretation of the mathematical result of the Legendre expansion. 


\section{Acknowledgments}

The authors wish to thank Dr M A Bratescu and Mr Y Suda of Hokkaido University for their interest and helpful discussions.

\section{References}

Engelhardt A G, Phelps A V and Risk C G 1964 Phys. Rev. 135 A1566-74

Garscadden A and Nagpal R 1995 Plasma Sources Sci. Technol. 4 268-80

Holstein T 1946 Phys. Rev. 70 367-84

Makabe T and Mori T 1982 J. Phys. D: Appl. Phys. 15 1395-402

Ohmori Y, Shimozuma M and Tagashira H 1988 J. Phys. D: Appl. Phys. 21 724-9

Penetrante B M and Bardsley J N 1983 J. Appl. Phys. 54 6150-3

Pitchford L C and Phelps A V 1982 Phys. Rev. A 25 540-54

Slavík J and Colonna G 1997 Plasma Chem. Plasma Processing 17 305-14

Sugawara H, Sakai Y, Tagashira H and Kitamori K 1998 J. Phys. D: Appl. Phys. 31 319-27

Sugawara H, Tagashira H and Sakai Y 1997 J. Phys. D: Appl. Phys. 30 368-73

Suzuki T, Fukuyama T and Itoh H 2001 Trans. Inst. Electr. Eng. Japan 121-A 256-62

Taniguchi T, Tagashira H and Sakai Y 1978 J. Phys. D: Appl. Phys. 11 1757-68 


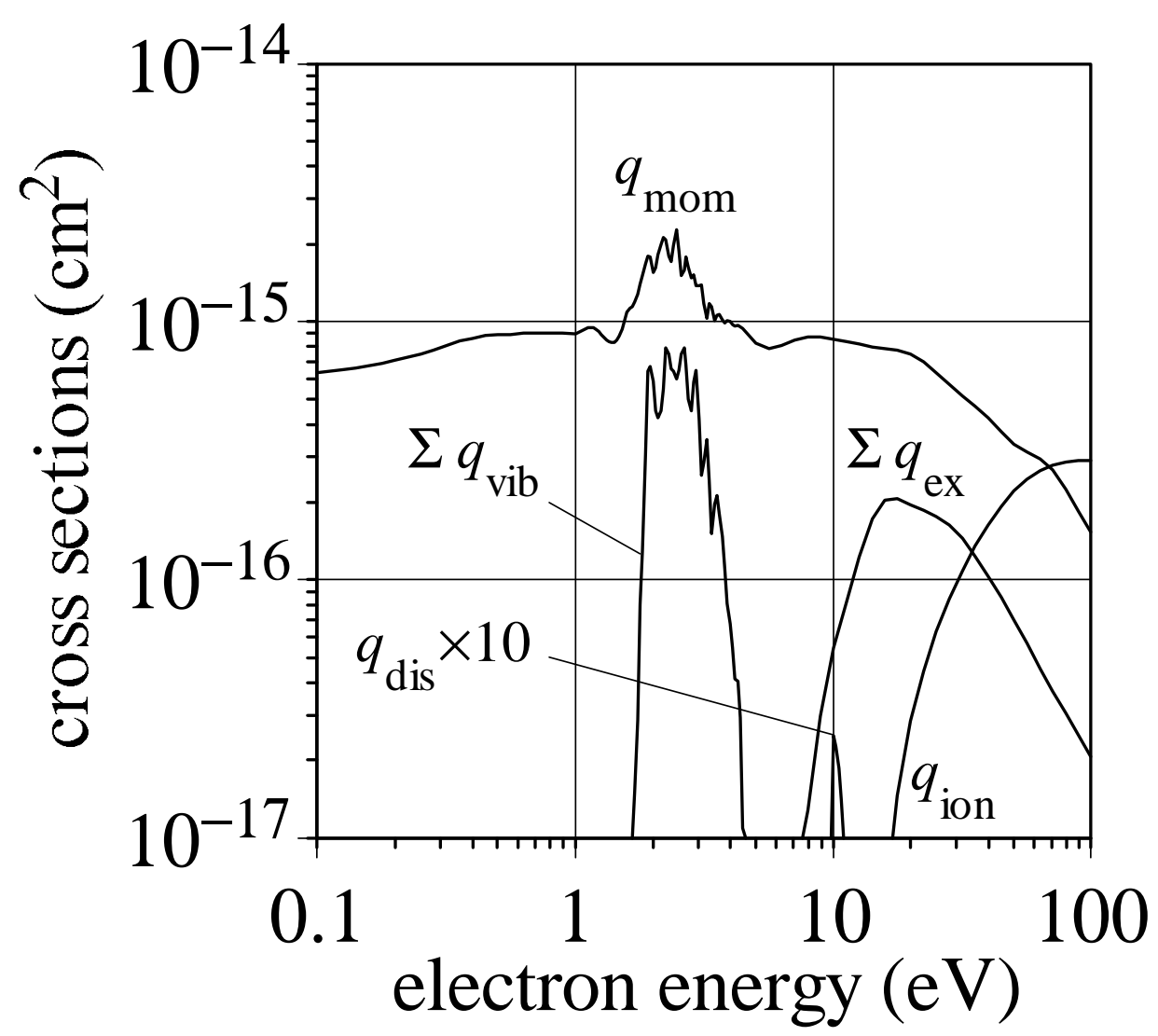

Figure 1. The electron collision cross sections of $\mathrm{N}_{2}$ (Ohmori et al 1988). 


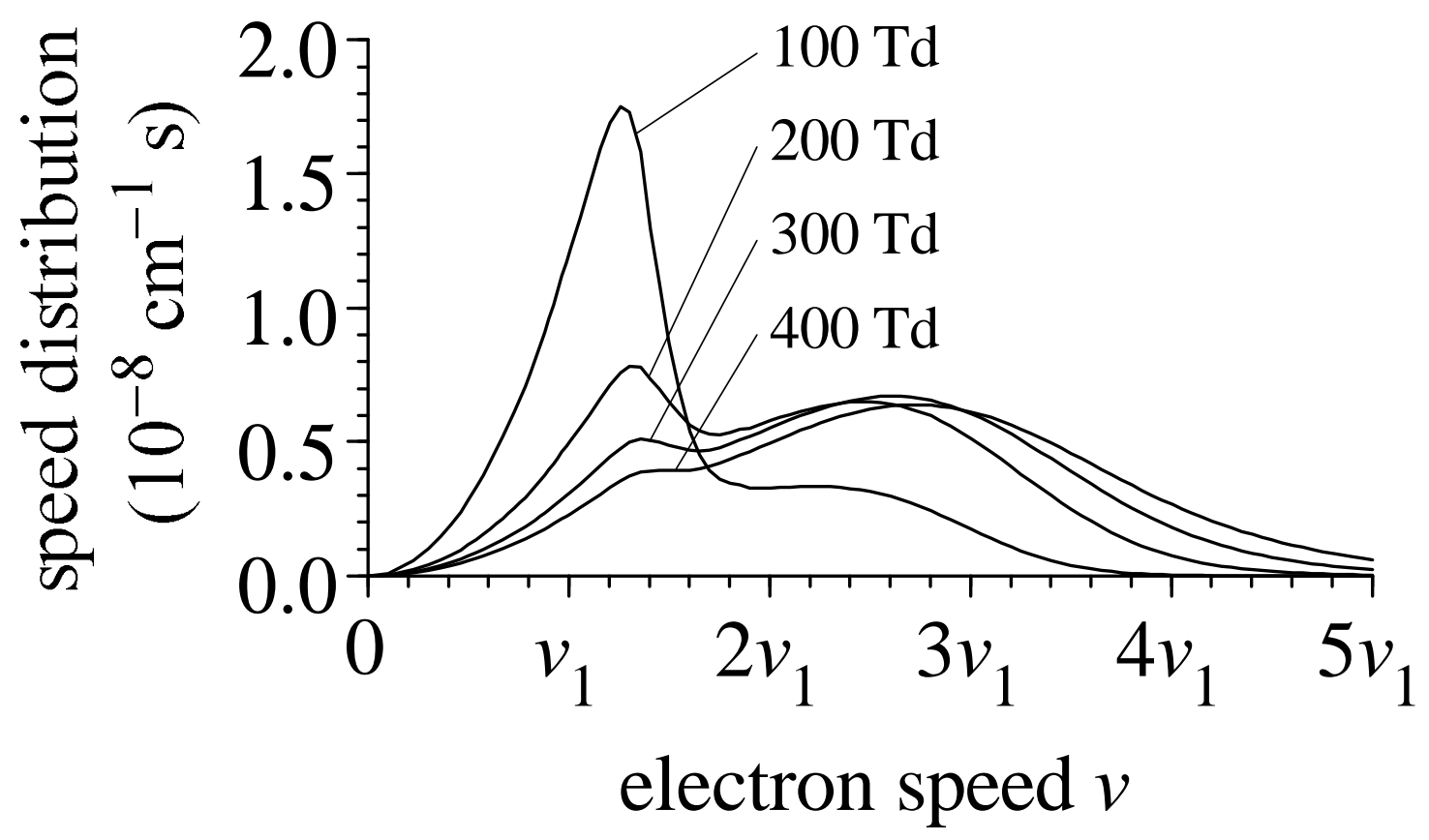

Figure 2. The electron speed distribution $f(v)$ in $\mathrm{N}_{2}$ calculated by PM at 100-400 Td. 

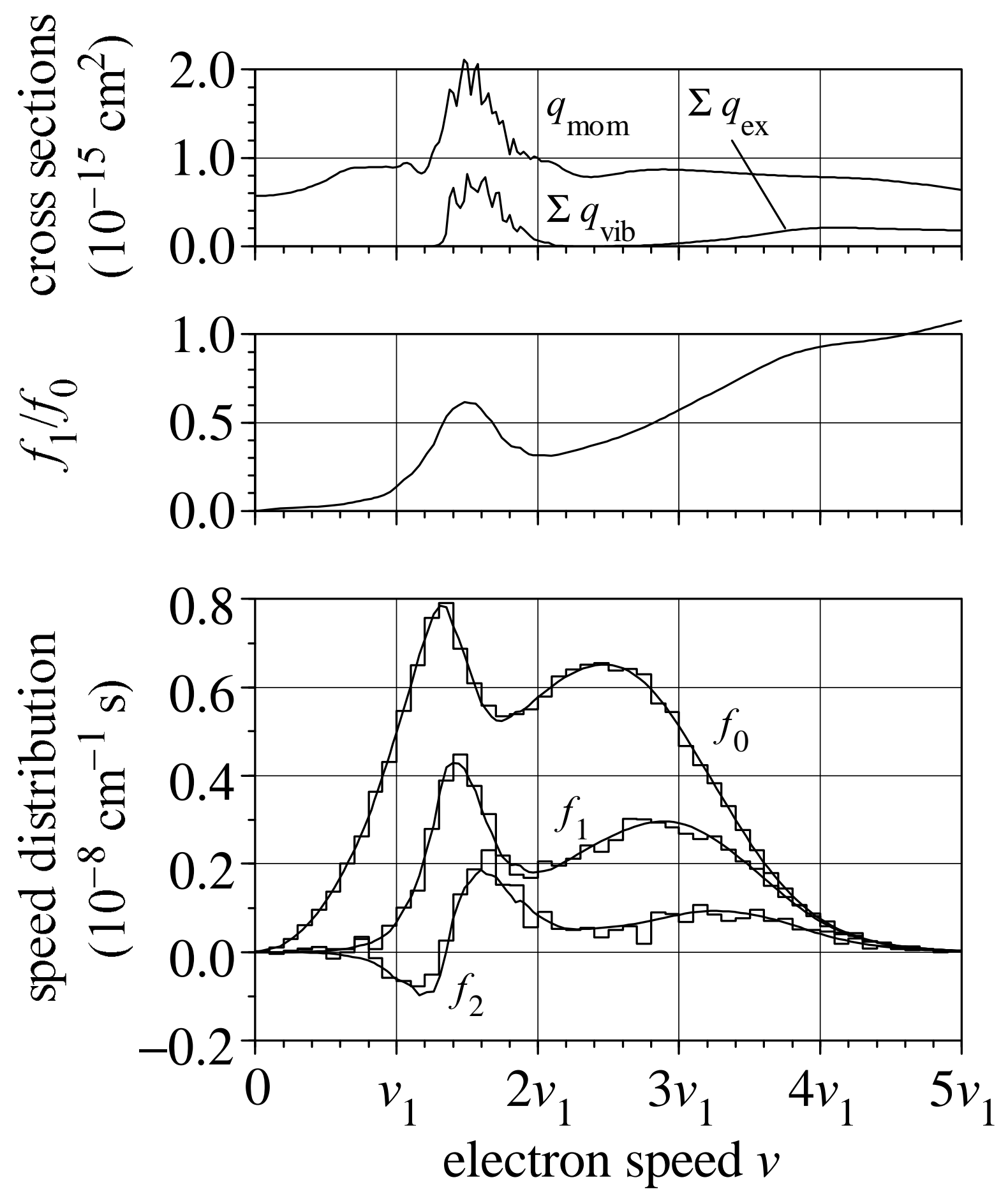

Figure 3. The Legendre polynomial expansion terms $f_{n}(v)(n=0,1,2)$ of the EVDF in $\mathrm{N}_{2}$ at 200 Td. Solid curve, PM; and histogram, MCS. The ratio $f_{1} / f_{0}$ representing the anisotropy of the EVDF and cross sections are shown together for comparison. 


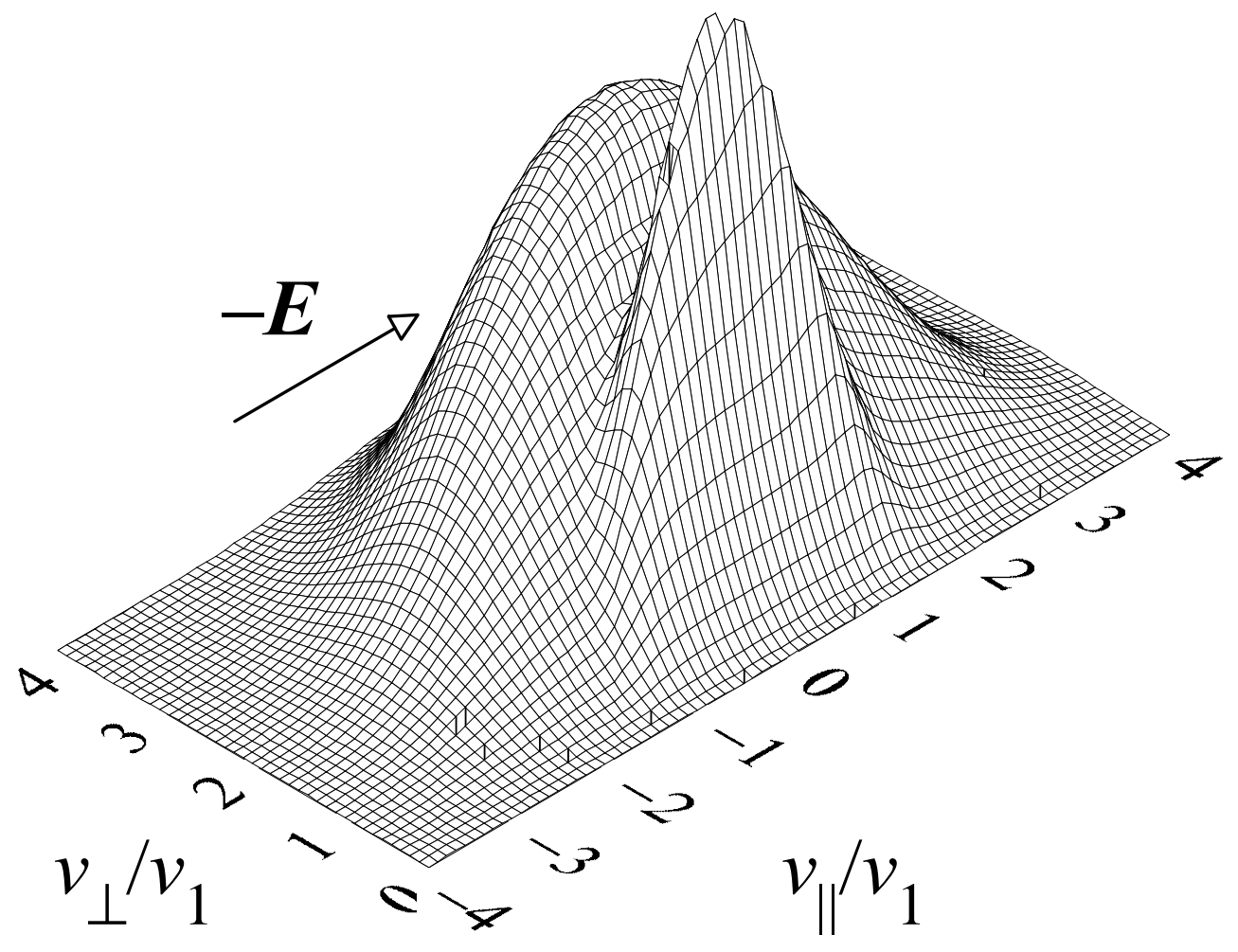

Figure 4. A two-component form of the EVDF, $f\left(v_{\|}, v_{\perp}\right)$, in $\mathrm{N}_{2}$ at $200 \mathrm{Td}$. 


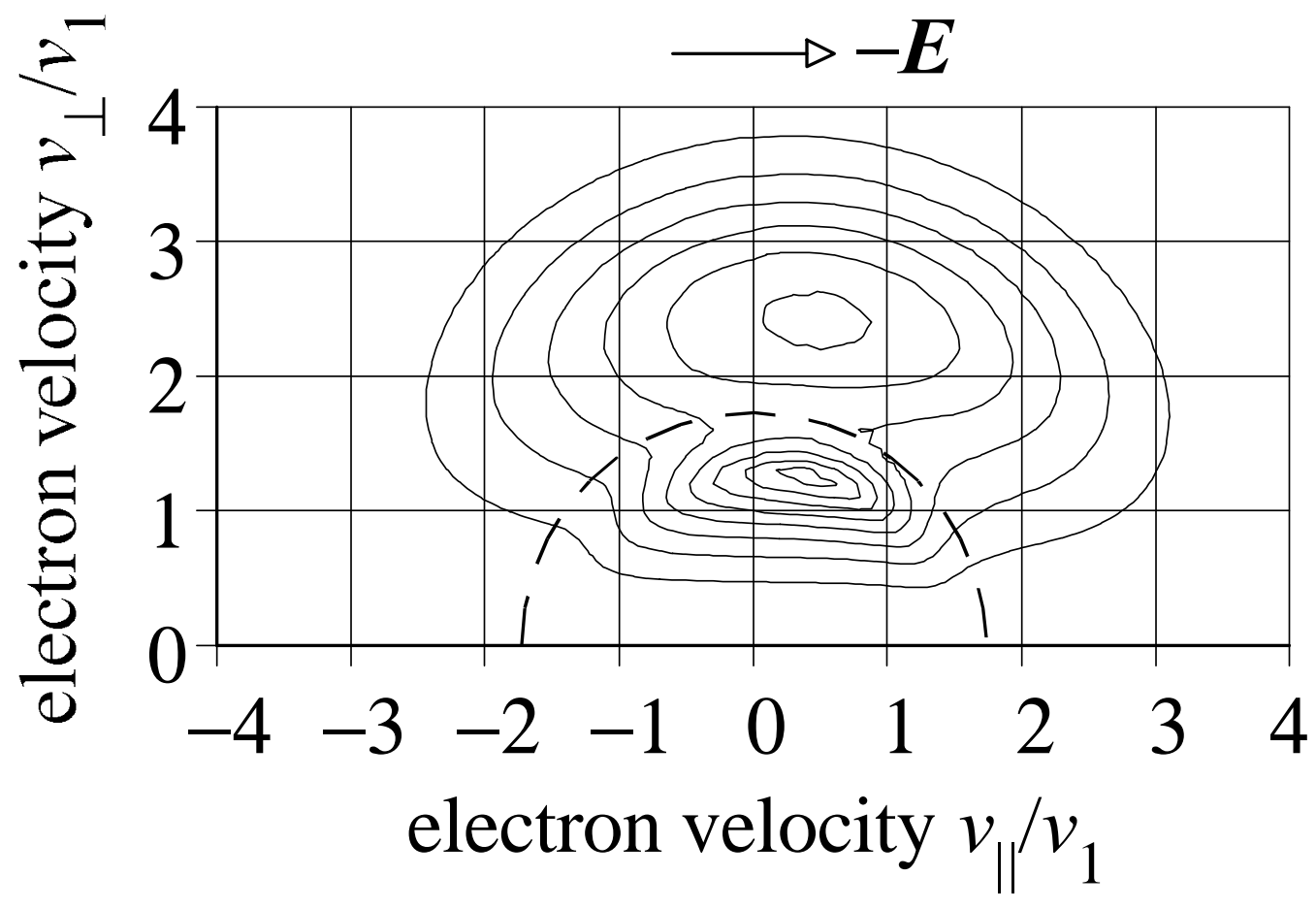

Figure 5. Contour plot of $f\left(v_{\|}, v_{\perp}\right)$ in $\mathrm{N}_{2}$ at $200 \mathrm{Td}$. The broken semicircle indicates the line of $\epsilon=3 \mathrm{eV}$. 


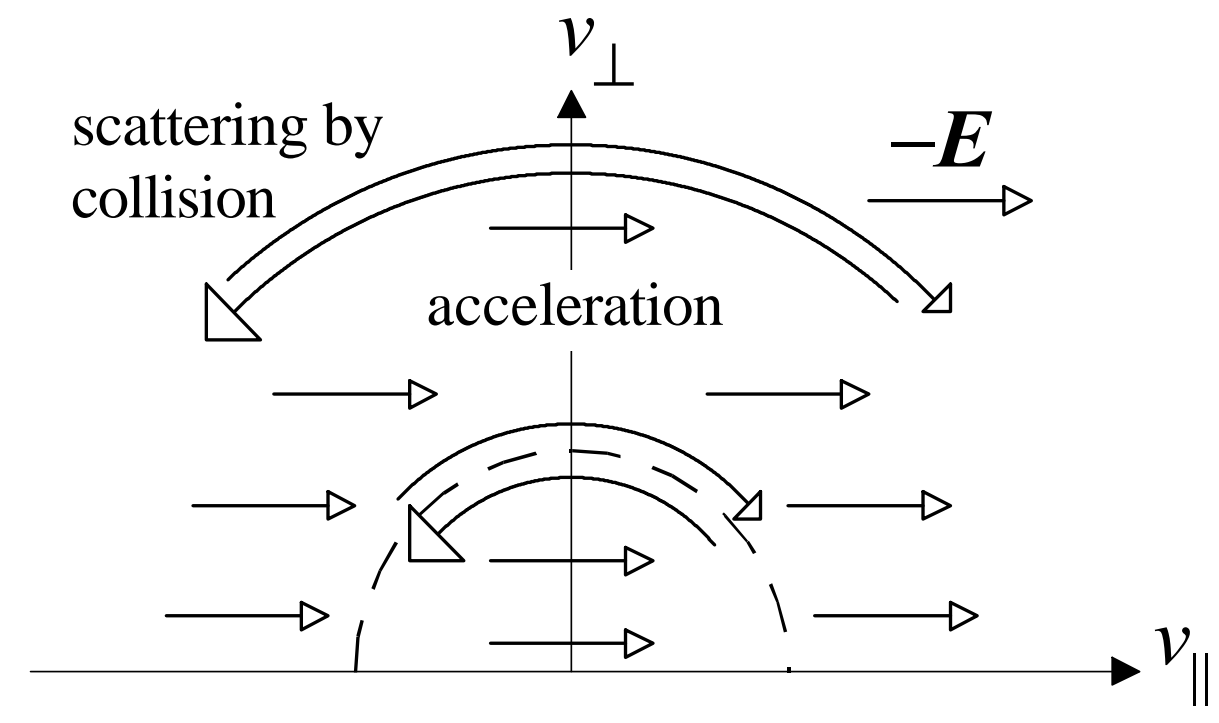

Figure 6. Schematic of electron flow in velocity space. The broken semicircle indicates the barrier originating in the $q_{\text {mom }}$ and $q_{\text {vib }}$ peaks. 


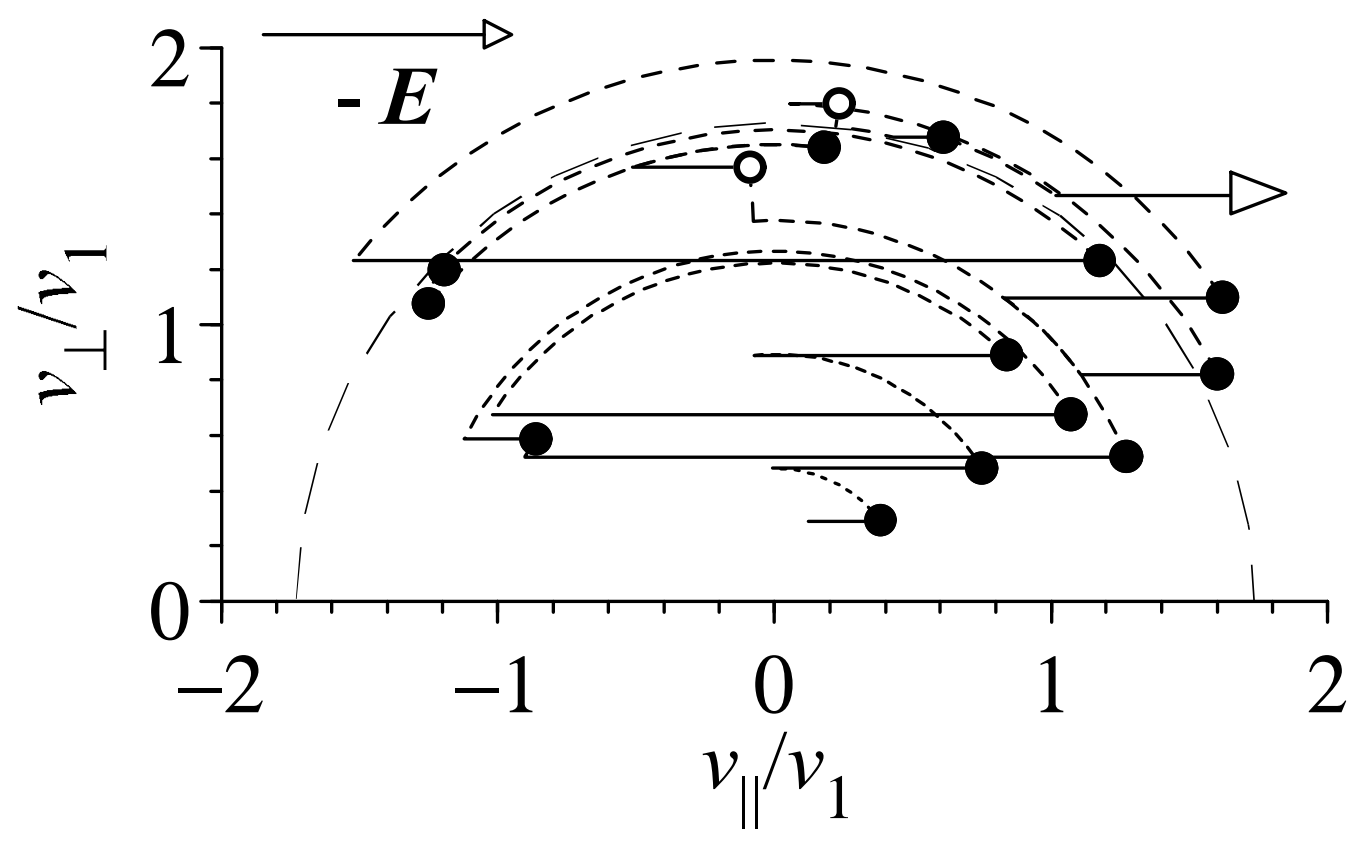

Figure 7. A single electron motion in $\mathrm{N}_{2}$ observed in velocity space. Solid line, electron flight; broken arc, scattering at collision; full circle, momentum transfer collision; and open circle, vibrational excitation collision. The broken semicircle indicates the line of $\epsilon=3 \mathrm{eV}$. 


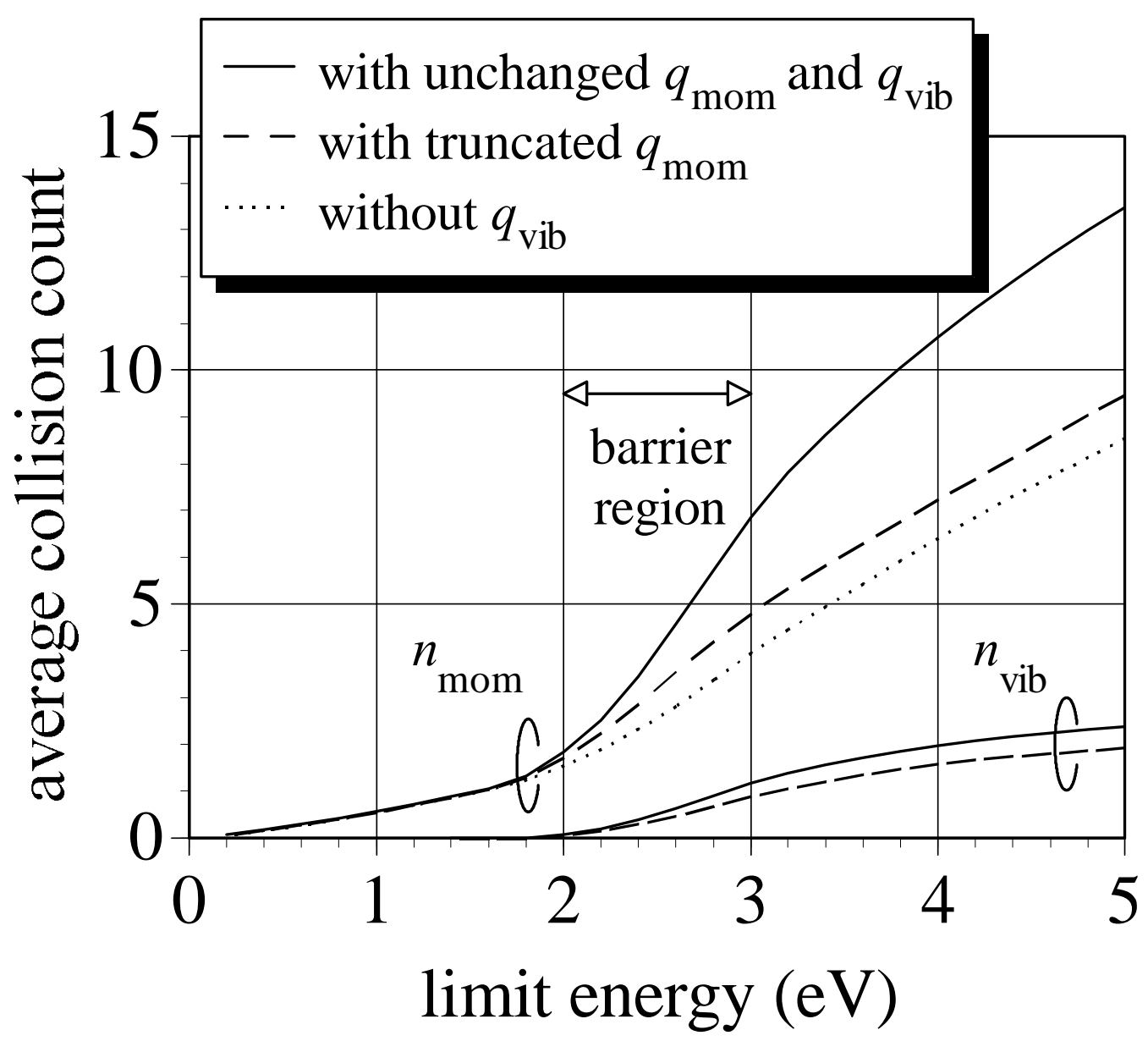

Figure 8. The average numbers of momentum transfer and vibrational excitation collisions, $n_{\mathrm{vib}}$ and $n_{\text {mom }}$, that an electron starting its flight with $\epsilon=0.1 \mathrm{eV}$ experiences until $\epsilon$ exceeds a limit energy. $E / N=200 \mathrm{Td}$. Solid curve, calculated with the cross section set of $\mathrm{N}_{2}$ from Ohmori et al (1988); broken curve, with $q_{\text {mom }}$ truncated at $10^{-15} \mathrm{~cm}^{2}$; and dotted curve, without $q_{\mathrm{vib}}$. 

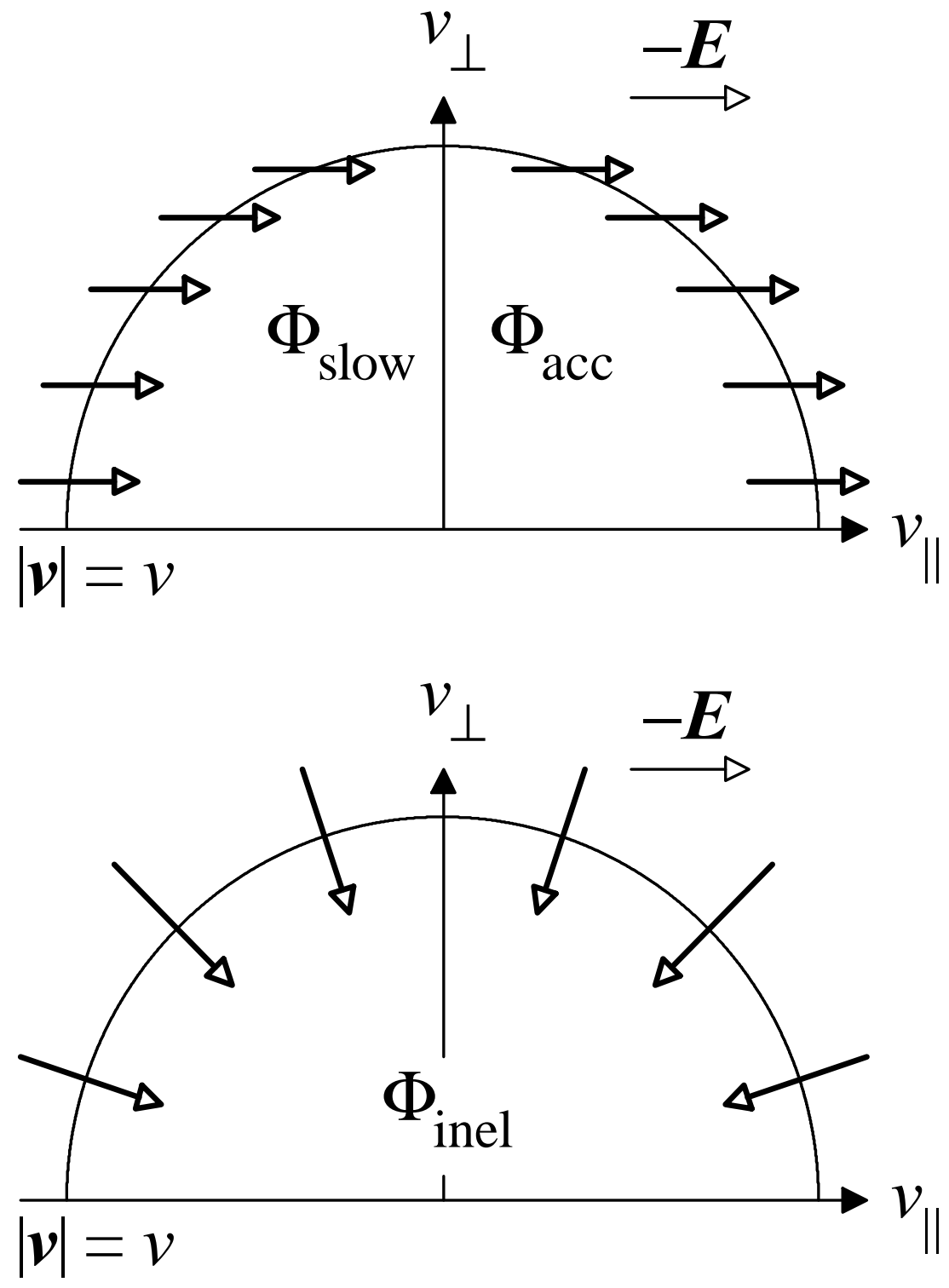

Figure 9. Schematic of the electron flow across a boundary at $v$ in velocity space; acceleration flow $\Phi_{\text {acc }}$, slowdown flow $\Phi_{\text {slow }}$, and transfer due to inelastic collision $\Phi_{\text {inel }}$. 


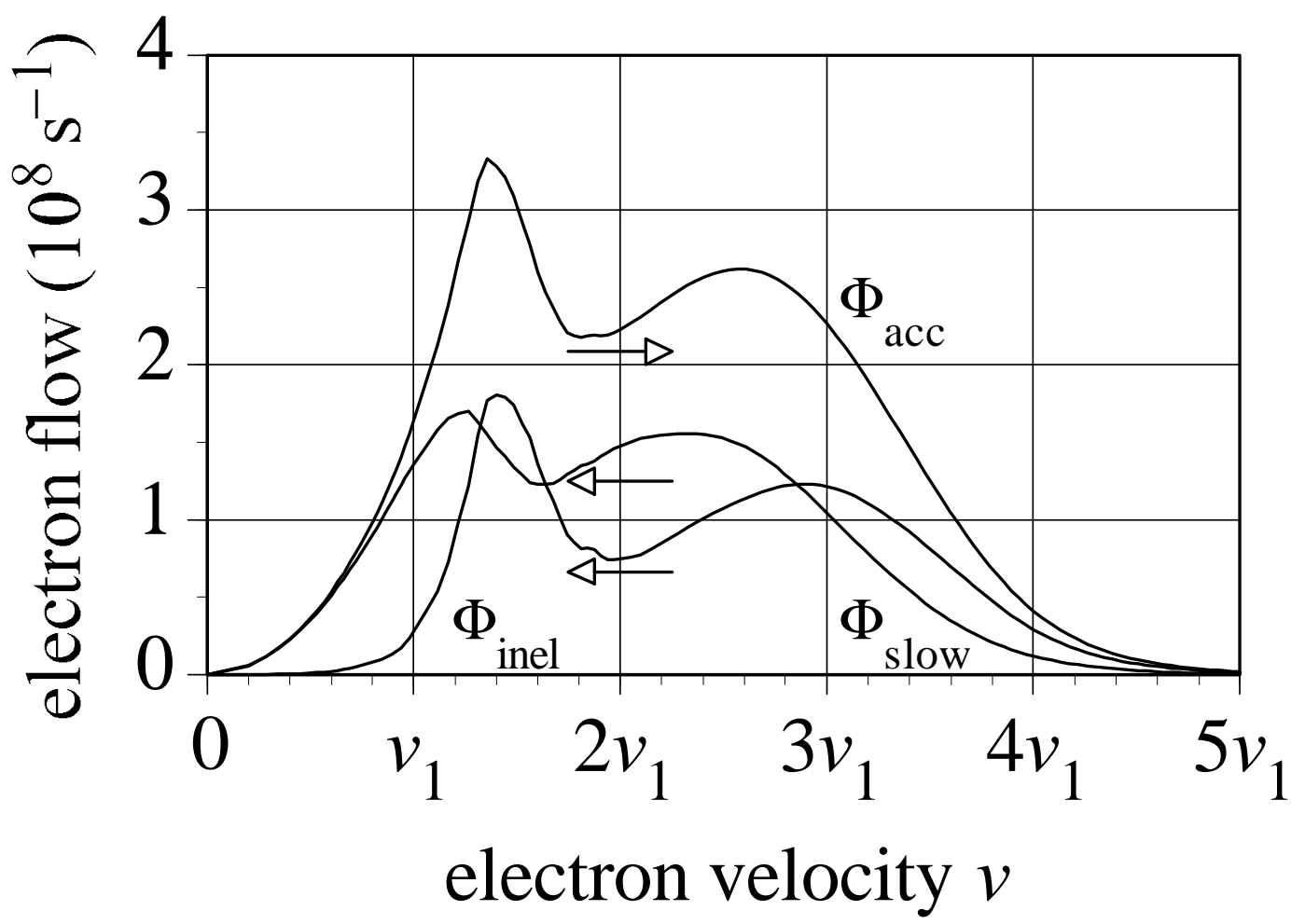

Figure 10. Electron flow across $v$ in velocity space. $\Phi_{\text {acc }}$, the acceleration flow; $\Phi_{\text {slow }}$, the slowdown flow; and $\Phi_{\text {inel }}$, the electron transfer due to inelastic collision. $E / N=200 \mathrm{Td}$. 


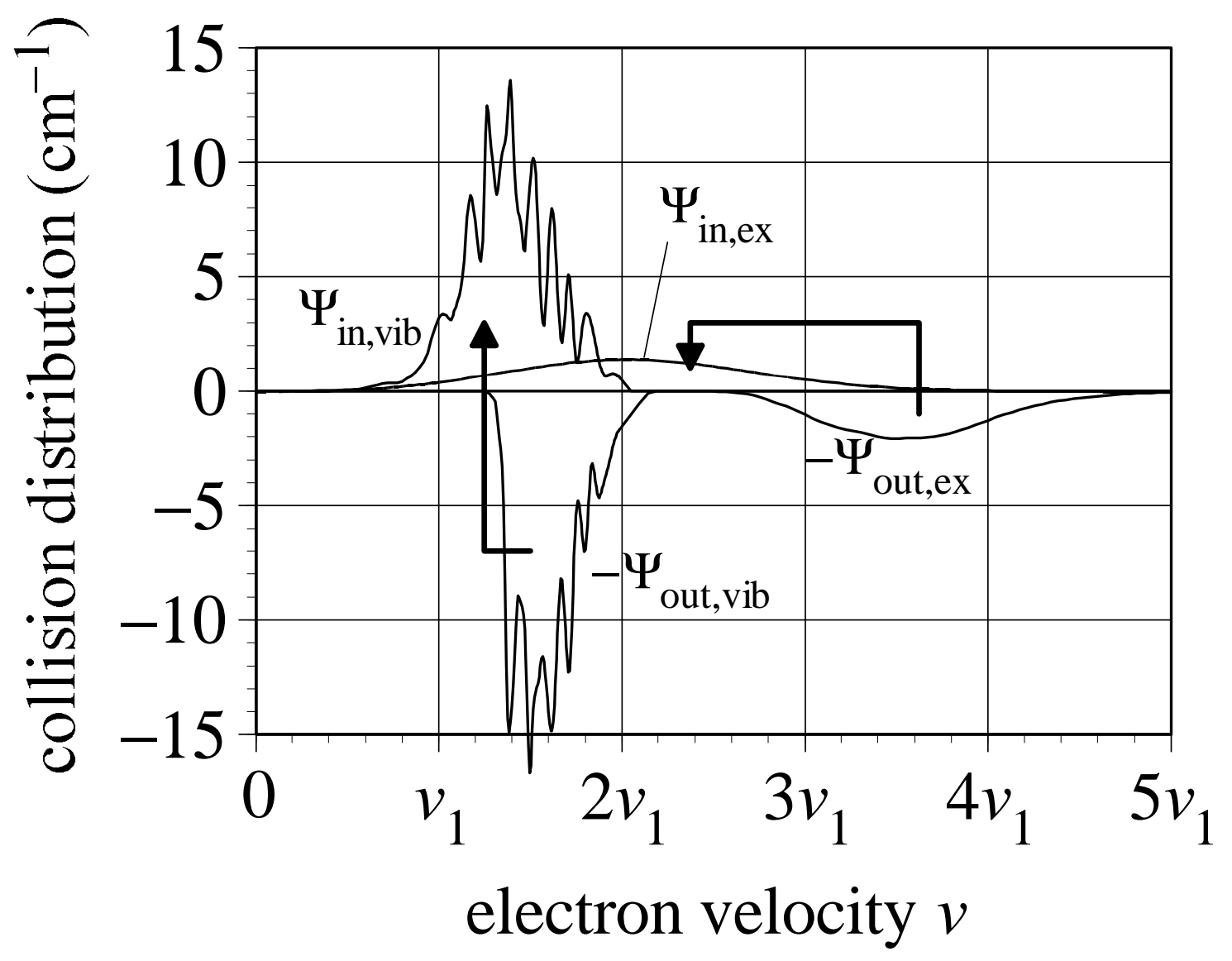

Figure 11. Distributions of $v^{\prime}$ and $v$ for electron velocity transition $v^{\prime} \rightarrow v$ at vibrational and electronic excitations in $\mathrm{N}_{2}$ at $200 \mathrm{Td}$. $\Psi \mathrm{d} v$ gives the number of transitions per unit time in $\mathrm{d} v$. 